\title{
Postpartum fatal cerebral vein thrombosis: A case report and review
}

\author{
Marina Kontogiorgi ${ }^{1}$, Vasiliki Kalodimou ${ }^{2}$, Spyros Kollias ${ }^{1}$, Demetrios Exarchos ${ }^{3}$, Serafim Nanas ${ }^{1}$, \\ Abraham Ghiatas $^{2}$, Christina Routsi ${ }^{{ }^{*}}$
}

${ }^{1}$ Critical Care Department, Medical School of Athens University, Evangelismos Hospital, Athens, Greece

${ }^{2}$ IASO Maternity Hospital, Athens, Greece

${ }^{3}$ Department of Radiology, Evangelismos Hospital, Athens, Greece

Email: ${ }^{*}$ croutsi@hotmail.com

Received 15 November 2011; revised 7 December 2011; accepted 16 January 2012

\begin{abstract}
Background: Cerebral vein thrombosis, is an emergent condition presenting with a variety of symptoms ranging from isolated headache to profound coma. Case Presentation: We present the case of a previously healthy young woman on the 18th postpartum day admitted into our hospital with a persistent headache for three days followed by seizures and coma. Magnetic Resonance Angiography revealed superior sagittal sinus and right transverse sinus thrombosis. Despite supportive measures and anticoagulation, the patient died because of uncontrolled increase in intracranial pressure. Conclusion: The possibility of cerebral vein thrombosis should be considered in all women with brain dysfunction during the puerperium.
\end{abstract}

Keywords: Cerebral Vein Thrombosis; Headache;

Seizure; Magnetic Resonance Angiography;

Venography; Puerperium

\section{INTRODUCTION}

Cerebral vein thrombosis, although relatively rare, is an emergent condition that can present itself with a variety of symptoms ranging from isolated headache to profound coma [1-3]. Major predisposing factors are hypercoagulable states including acquired prothrombotic conditions such as pregnancy and puerperium [1-5]. The diagnosis needs a high index of suspicion and the use of neuroimaging techniques. A fatal case of cerebral vein thrombosis is described in a young woman postpartum, presenting with persistent headache followed by seizures and coma, misdiagnosed as intracranial hemorrhage.

\section{CASE REPORT}

A 32-year-old woman was admitted to a local hospital

\footnotetext{
"Corresponding author.
}

with a 3-day history of severe and increasing headache. Previous medical history was unremarkable and she had a vaginal forceps assisted delivery 18 days prior. There was not a history of pregnancy-related hypertension. Soon after admission, she presented a generalized seizure and subsequent left hemiplegia, and she was removed to our tertiary-care hospital under anticonvulsant therapy. A computed tomography (CT) scan of the brain, performed without administration of contrast material, revealed a diffuse hypodence lesion in the right cortical sulci and subcortical regions with small foci of hemorrhage; the diagnosis of intracranial hemorrhage was made. On the third hospital day the patient became comatose, responding only to pain (Glasgow Coma Scale 8) and was transferred to the Intensive Care Unit (ICU) intubated, mechanically ventilated, and sedated with midazolam. The pupils were equal and reactive, the vital signs within normal range, the hematocrit was $27.8 \%$; the white-cell count was $13,440 / \mathrm{mm}^{3}$ with $91 \%$ neutrophils and the platelet count $198,000 / \mathrm{mm}^{3}$. Routine biochemical parameters were normal. Coagulation profile including prothrombin time, partial thromboplastine time, protein $\mathrm{C}$, protein $\mathrm{S}$ and antiphospholipid antibodies were within normal limits. The chest radiograph was unremarkable. Transesophageal echocardiography did not reveal any abnormal finding. Repeated CT scan of the brain showed the hemorrhagic infract and generalized edema (Figure 1). Magnetic resonance imaging (MRI) and MR angiography revealed absence of flow signal in the superior sagittal sinus and right transverse sinus (Figure 2); the diagnosis of cerebral vein thrombosis, resulting in hemorrhagic infraction of the right frontal and parietal lobe was made. Intravenous unfrarctionated heparin was immediately started. The presence of an increased intracranial pressure (ICP) was confirmed with the placement of an intracranial catheter. Despite supportive measures including sedation (midazolam), anti-epileptic (phenyntoin) and anti-edematous (mannitol and hypertonic solutions) 


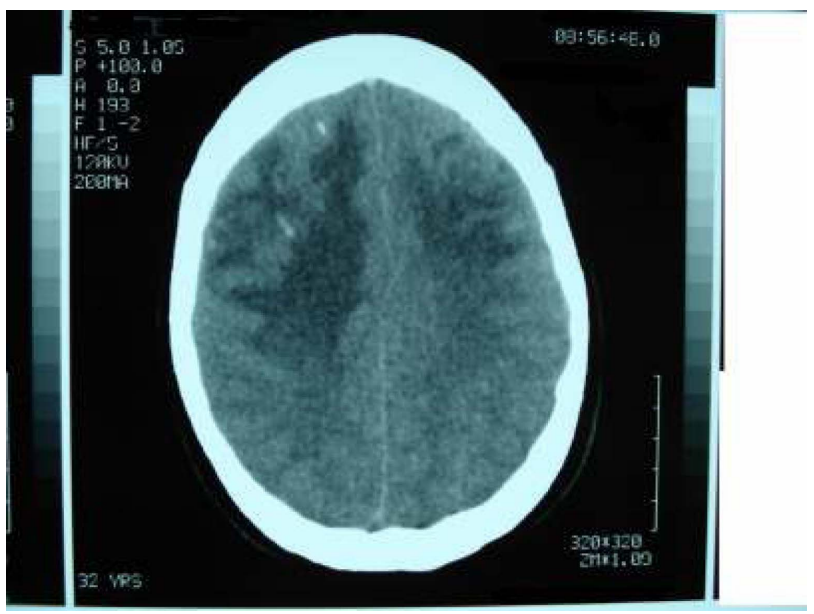

Figure 1. CT axial image. Diffuse hypodense lesion in cortical solci and subcortical regions. An intracranial catheter is present in the right frontal lobe. Small foci of hemorrhage present.

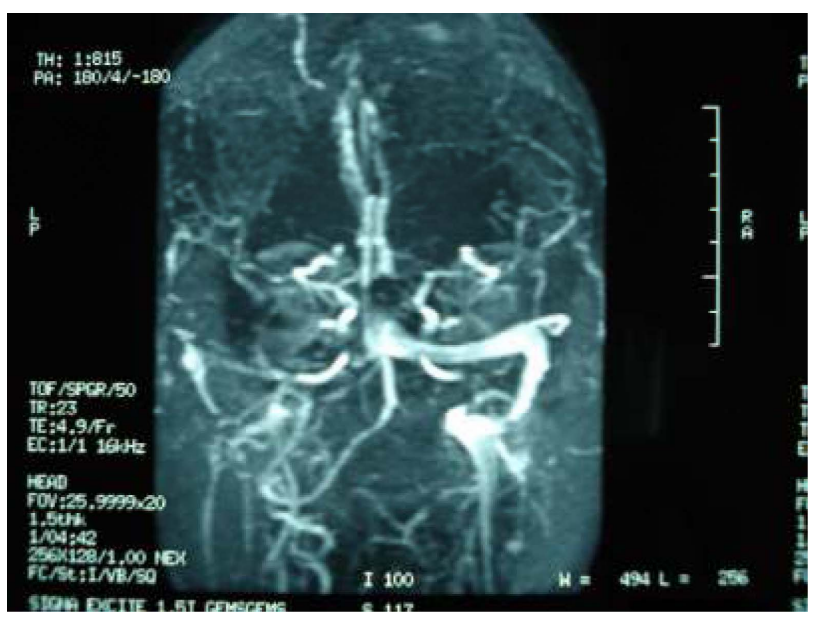

Figure 2. 3D Time-of-Flight MR angiography, coronal view. Absent flow signal in superior sagittal sinus and right transverse sinus.

treatment, and finally barbiturate coma, the ICP continued to increase and on the third ICU day a decompressive craniotomy was performed. However, the neurologic status deteriorated with signs of cerebral herniation. The presence of brain death was diagnosed and the patient died four days later.

\section{DISCUSSION}

Cerebral vein thrombosis is an infrequent, but always a serious and emergency entity. There are several recognized causes or predisposing conditions [1-3]. Among the major risk factors for cerebral vein thrombosis is a hypercoagulable state. In young women, the last trimester of pregnancy and especially the puerperium represent important risk factors for this condition with an incidence estimated about 12 cases per 100,000 deliveries. A prothrombic state in both pregnancy and puerperium has been implicated. Pregnancy-related hypertension and cesarean delivery further increase the risk [1-6]. These obstetrical causes along with the oral contraceptive use, give a significant female predominance among young adults with the diagnosis of cerebral vein thrombosis (70\% - 80\%) [1]. Additional risk factors were not identified in our patient; the only predisposing factor seems to be the postpartum period she was running.

Cerebral vein thrombosis is characterized by a wide variety of symptoms, relatively nonspecific. Headache is the most frequent and often the earliest symptom. It can be of any grade of severity; usually is global, persistent, and has an acute onset. When it occurs in the absence of any other neurologic sign, as in the present case, it raises great diagnostic difficulties. Seizures, focal or generalized, occur in about $40 \%$ of the patients. Other clinical manifestations depend on the location of cerebral lesions and/or the development of raised intracranial pressure. They include papilledema, cranial nerve palsies, unilateral hemispheric symptoms such as hemiparesis or aphasia, various cognitive or psychiatric disturbances, and impaired mental status [1-6].

The development of new neuroimaging diagnostic techniques have greatly contributed to the increasingly recognition of this entity. The diagnosis can be suspected from brain CT scan, although sometimes it is normal, and be confirmed with conventional angiography or CT angiography or MRI combined with MR angiography of the brain, the latter thought to be the diagnostic procedure of choice [7]. The diffuse hypotense lesion found on admission CT scan in this patient was in fact a venous infraction accompanied by hemorrhage, and should serve as an indirect sign of cerebral vein thrombosis, before the direct findings of MR angiography became available.

Most patients improve with the appropriate management. Current therapy consists of aggressive anticoagulation with heparin combined with lowering intracranial pressure maneuvers [8]. In a retrospective study the final outcome has been considered good in $80 \%$ of patients with obstetric etiologies, compared with a $58 \%$ of patients with other causes [4]. An even better clinical outcome for cases related to pregnancy and the puerperium has been reported recently [9]. However, cerebral vein thrombosis remains a potentially life-threatening complication. Markedly impaired consciousness upon presentation, hemorrhage found at the brain CT scan on admission, delay in diagnosis and therefore in the treatment, are poor prognostic factors [1-3]. In our patient all these three factors predominated. However, the lack of a required high index of suspicion probably due to the rare incidence of this entity, contributed to initial misdiagnosis as a case of intracranial hemorrhage and a delay in the carrying out of a MR angiography. Delay in diagnosis is 
also present in several of the reported cases in the literature, indicating the need for a reminder [10-12].

\section{CONCLUSION}

Cerebral vein thrombosis in the puerperium is a life threatening complication. Given the considerable potential for full recovery in early diagnosis and the lethal outcome in unrecognized and untreated cases, the possibility of cerebral vein thrombosis should be considered as an emergency in women presenting neurological symptoms, even only headache, during puerperium.

\section{REFERENCES}

[1] Stam, J. (2005) Thrombosis of the cerebral veins and sinuses. The New England Journal of Medicine, 352, 17911798. doi:10.1056/NEJMra042354

[2] Bousser, M.G. (2000) Cerebral venous thrombosis: Diagnosis and management. Journal of Neurology, 247, 252-258. doi:10.1007/s004150050579

[3] Crassard, I. and Bousser, M.G. (2004) Cerebral venous thrombosis. Journal of Neuro-Ophthalmology, 24, 156163. doi:10.1097/00041327-200406000-00011

[4] Cantou, C. and Barinagarrementeria, F. (1993) Cerebral venous thrombosis associated with pregnancy and puerperium: Review of 67 cases. Stroke, 24, 1880-1884. doi:10.1161/01.STR.24.12.1880

[5] Mas, J.L. and Lamy, C. (1998) Stroke in pregnancy and the puerperium. Journal of Neurology, 245, 305-313. doi:10.1007/s004150050224
[6] Ferro, J.M., Canhao, P., Starn, J., Bousser, M.G. and Barinagarrementeria, F. (2004) Prognosis of cerebral vein and dural sinus thrombosis: results of the international study on cerebral vein and dural sinus thrombosis (ISCVI). Stroke, 35, 664-670. doi:10.1161/01.STR.0000117571.76197.26

[7] Ozsvath, R.R., Casey, S.O., Lustrin, E.S., Alberico, R.A., Hassankhani, H. and Patel, M. (1997) Cerebral venography: Comparison of CT and MR projection venography. American Journal of Roentgenology, 169, 1699-1707.

[8] Einhaupl, K., Stam, J., Bousser, M.G., De Bruijn, S.F.T.M., Ferro, J.M., Martinelli, M. and Masuhr, F. (2010) EFNS guideline on the treatment of cerebral venous and sinus thrombosis in adult patients. European Journal of Neurology, 17, 1229-1235. doi:10.1111/j.1468-1331.2010.03011.x

[9] Lanska, D.J. and Kryscio, R.J. (2000) Risk factors for peripartum and postpartum stroke and intracranial venous thrombosis. Stoke, 31, 1274-1282.

[10] Chan, K.H., Cheung, R.T.F., Liu, W.M., Mak, W. and Ho, S.L. (2005) Cerebral venous thrombosis in a gentleman presenting with fever, convulsion and frontotemporal haemorrhages. Journal of Clinical Neuroscience, 12, 186188. doi:10.1016/j.jocn.2004.03.013

[11] Pannke, T.S. (1991) Cerebral dural sinus thrombosis. Annals of Emergency Medicine, 20, 813-816. doi:10.1016/S0196-0644(05)80849-2

[12] Nazziola, E. and Elkind, M.S. (2003) Dural sinus thrombosis presenting three months postpartum. Annals of Emergency Medicine, 42, 592-595. doi:10.1067/S0196-0644(03)00389-5 